\title{
Percepção de Oportunidades de Aprendizado de Funcionários Técnico-Administrativos no Âmbito Universitário
}

\author{
Juliana de Araújo Germano ${ }^{1}$ \\ 1Universidade Católica de Pelotas, RS, Brasil. \\ Cainá Corrêa do Amaral ${ }^{1}$ \\ 1Universidade Católica de Pelotas, RS, Brasil. \\ Tiago Fernandez Garcia ${ }^{1}$ \\ 1 Universidade Católica de Pelotas, RS, Brasil. \\ Geovanna Peter Corrêa ${ }^{1}$
}

\author{
1Universidade Católica de Pelotas, RS, Brasil. \\ Camila Perelló Ferrúa ${ }^{1}$ \\ 1Universidade Católica de Pelotas, RS, Brasil. \\ Luciana de Ávila Quevedo ${ }^{1}$ \\ 1Universidade Católica de Pelotas, RS, Brasil. \\ Fernanda Nedel ${ }^{1}$ \\ 1Universidade Católica de Pelotas, RS, Brasil.
}

\begin{abstract}
Resumo: Este estudo tem por objetivo identificar a percepção de oportunidades de aprendizagem e sua relação com o estilo de liderança exercido pelos funcionários técnicoadministrativos no âmbito universitário, a intenção de rotatividade e capital psicológico no trabalho. Foi realizado um estudo transversal, com 102 funcionários técnico-administrativos, por meio de um questionário de autopreenchimento contendo dados referentes a idade, escolaridade, tempo de serviço na instituição e se o respondente exercia um cargo de liderança. Foram aplicadas escalas relacionadas à percepção de oportunidades de aprendizagem, avaliação do estilo gerencial, intenção de rotatividade e capital psicológico no trabalho. Os resultados revelam que os funcionários identificam oportunidades de aprendizado e a presença de três estilos gerenciais no âmbito universitário. Também se observou que os funcionários se identificam como capitais psicológicos no trabalho e possuem baixa intenção de rotatividade. Assim, este trabalho buscou ampliar a literatura ainda escassa envolvendo funcionários técnico-administrativos em âmbito universitário.
\end{abstract}

Palavras-chave: Aprendizagem social, Comportamento social, Rotatividade de pessoal.

\section{Perception Learning Opportunities of Technical- Administrative Staff in the University Scope}

\begin{abstract}
This study aimed to identify the perception of learning opportunities and its association with the leadership style of the administrative-technical staff within the university scope, the turnover intention, and the psychological capital at work. For that, this cross-sectional study was conducted with data on age, education level, length of service in the institution, and leadership position of 102 administrative and technical employees, collected by means of a selfadministered questionnaire. Perceived learning opportunities, management style, turnover intention, and psychological capital at work were evaluated using scales. The results show that the administrative-technical staff identifies the three management styles and learning opportunities within the university scope. Moreover, they identify themselves as psychological capitals at work and possess low turnover intention. This study is expected to expand the (still scarce) literature on administrative and technical staff in the university context.
\end{abstract}

Keywords: Social learning, Social behavior, Staff turnover. 


\title{
Percepción del Personal Técnico y Administrativo sobre las Oportunidades de Aprendizaje en las Universidades
}

\begin{abstract}
Resumen: Este estudio tuvo como objetivo identificar las oportunidades de aprendizaje y su relación con el estilo de liderazgo adoptado por el personal técnico y administrativo en las universidades, la intención de rotación y el capital psicológico en el trabajo. Este es un estudio transversal realizado con 102 personales técnicos y administrativos mediante la aplicación de un cuestionario de autoinforme con datos sobre la edad, la educación, el tiempo de servicio en la institución y si ejercían cargo de liderazgo. Se aplicaron las escalas en cuanto a la percepción de las oportunidades de aprendizaje, la evaluación del estilo de gestión, la intención de rotación y el capital psicológico en el trabajo. Los resultados muestran que el personal identifica oportunidades de aprendizaje y la presencia de tres estilos de gestión en la universidad. También se observó que ellos se identifican como capital psicológico en el trabajo y tienen la intención de baja rotación. Por lo tanto, este estudio trató de ampliar la literatura todavía escasa, con la participación del personal técnico y administrativo en el ámbito universitario.
\end{abstract}

Palabras clave: Aprendizaje social, Comportamiento social, Rotación de personal.

\section{Introdução}

O processo de aprendizagem dentro das empresas possui um papel estratégico para a expansão do negócio e adaptação permanente às necessidades dos clientes e do mercado. Para o desenvolvimento de novas competências organizacionais, é preciso investir em pessoas e processos por meio de capacitação, disseminação de informação, troca de experiências, definição e compartilhamento da forma de realizar as tarefas na instituição. Além de as empresas precisarem de inovação para se tornar cada vez mais competitivas, há também a necessidade dos indivíduos pela busca do desenvolvimento e, como consequência, o crescimento profissional (Luthans, Avey, Avolio, \& Peterson, 2010; Veloso, Silva, Silva, \& Caetano, 2015).

Para que o processo de melhoria contínua se instale nas organizações, a aprendizagem deve ser o foco da instituição. Isto é, aprendizagem organizacional, gerencial, comportamental e de novas rotinas de trabalho, mediante o desenvolvimento de competências, proporcionando maior conhecimento, habilidades para realizar as tarefas e atitudes frente a cada situação, gerando, dessa forma, novas competências individuais, sistêmicas e diferenciais no desempenho (Picoli \& Takahashi, 2016). As ações de Treinamento, Desenvolvimento e Educação (TD\&E) tornaram-se um tema que vem recebendo atenção de pesquisadores e acadêmicos nos últimos anos. Segundo Santos
Filho e Mourão (2011), o processo de aprendizagem é permanente e está associado às possibilidades de crescimento na carreira profissional. Além disso, é intrínseco ao ser humano e irá acompanhar o indivíduo por toda a vida. Embora exista uma vasta literatura sobre os impactos do treinamento no trabalho (análise sobre as medidas, motivações, benefícios, entre outros) (Luthans et al., 2010; Veloso et al., 2015; Picoli \& Takahashi, 2016; Santos Filho \& Mourão, 2011), os conhecimentos adquiridos transpassam o ambiente organizacional e adaptam-se aos diversos contextos sociais (Lacerda \& Abbad, 2003).

Há também outros fatores que influenciam os processos de aprendizagem nas organizações, como a cultura da empresa, a motivação e o comprometimento dos funcionários. No entanto, o fator liderança destaca-se em relação aos demais. Ele será definido como uma variável de identificação de mecanismos de aprendizagem nas instituições (Melo, 2004). Além disso, o pressuposto básico é de que a produtividade, a qualidade e a motivação do funcionário perpassam pelo modo comportamental do gestor e por seu exemplo diário (Melo, 2004). A liderança nas organizações possui a responsabilidade técnica de fazer com que o resultado esperado seja atingido pelo setor, por meio de sua equipe. Para isso, utiliza de suas competências: conhecimento técnico sobre a área de atuação, habilidades de influenciar pessoas e atitudes e exemplos 
frente às situações diárias. Durante o período em que responde pela área, uma das maiores responsabilidades do gestor é desempenhar o papel fundamental de incentivar o desenvolvimento do setor para superar desafios e, com isso, tornar-se aquele que impulsiona as pessoas, identificando potenciais e investindo recursos para proporcionar o crescimento (Coelho Jr., 2011). A fundamentação teórica utilizada na proposta deste estudo baseia-se na Psicologia Positiva, que identifica no indivíduo qualidades e virtudes humanas, promovendo seu funcionamento positivo frente às necessidades do ambiente organizacional, com o intuito de proporcionar, além da prosperidade econômica da organização, a saúde e o bem-estar de seus funcionários (Chiuzi, Siqueira, \& Martins, 2012).

Luthans, Youssef e Avolio (2007) propõem um novo olhar para a força de trabalho, dando ênfase ao trabalhador individual por meio do capital psicológico (CAP). De acordo com Siqueira (2014), o CAP contempla quatro capacidades psicológicas positivas: autoeficácia, otimismo, esperança e resiliência. Pesquisas realizadas por Avey, Luthans e Youssef (2010) concluíram que o capital psicológico no trabalho possui relação negativa com o cinismo organizacional, as intenções de rotatividade e comportamentos contraproducentes no local de trabalho, além de demonstrar relação positiva com comportamentos organizacionais de cidadania.

De acordo com Luthans et al. (2010), cada uma dessas capacidades psicológicas é mensurável, pode ser desenvolvida e tem impacto positivo sobre o desempenho do indivíduo em organizações. A partir desse conceito, despertou-se o interesse em não somente identificar os "capitais psicológicos no trabalho", mas também a relação com os gestores, se existe uma prevalência maior de CAP em setores específicos e se há relação com os estilos de liderança.

Em estudo realizado na Austrália por Perryer, Jordan, Firns e Travaglione (2010), foi identificado que os funcionários podem ser menos propensos a deixar a sua organização se há aumento de seus níveis de comprometimento afetivo em conjunto com um aumento do apoio fornecido pela organização. No mesmo estudo, os autores ressaltam que os gerentes podem influenciar ativamente os fatores que causam a intenção de rotatividade de empregados. No entanto, como o empregado ainda não saiu da organização, a compreensão da intenção de rotatividade torna-se uma informação de valor para os gestores. Portanto, estudos que contribuam para a compreensão das relações entre essas variáveis não são simplesmente de interesse teórico, mas também são de valor prático considerável para os gestores que serão, então, capazes de resolver esses fatores de forma preventiva e evitar problemas com os resultados do negócio.

De acordo com Carmo (2009), os atos dos empregados relacionados a absenteísmo e turnover (rotatividade) traduzem de alguma forma como o trabalhador se sente em relação à organização e a seu trabalho. Para o autor, conhecer as atitudes e opiniões dos empregados é antecipar algo sobre suas decisões e comportamentos futuros.

Portanto, este estudo teve como objetivo identificar a percepção de oportunidades de aprendizagem dos funcionários técnico-administrativos no âmbito universitário. Além disso, relaciona-se a variável primária (percepção de oportunidade de aprendizagem) com o estilo de liderança, a intenção de rotatividade e o capital psicológico no trabalho. Cabe salientar a escassez de trabalhos que centralizam essa discussão no âmbito dos funcionários técnico-administrativos em instituições de ensino superior, especialmente no que tange ao cenário brasileiro, destacando assim a relevância deste trabalho. Dessa forma, os resultados desta pesquisa podem ser úteis para direcionar as políticas de gestão de pessoas, revisar os processos de capacitação e desenvolvimento dos funcionários, orientar gestores em condutas para a retenção e identificação do capital psicológico no trabalho.

\section{Metodologia}

Trata-se de um estudo transversal com os funcionários técnico-administrativos de uma instituição de ensino superior privada do Brasil, com característica comunitária e filantrópica. Este trabalho foi aprovado pelo Comitê de Ética em Pesquisa, sob o protocolo número 48273015.0.0000.5339. Todos os participantes foram voluntários, concordaram em participar da pesquisa e assinaram um termo de consentimento livre e esclarecido.

\section{Participantes}

Estudo composto por 102 funcionários técnico-administrativos, o que representa $40 \%$ dos funcionários da instituição de ensino, por meio de amostra de conveniência, com no mínimo seis meses de relacionamento funcional com seus gestores imediatos. 
Os funcionários terceirizados ou que durante o período de aplicação dos questionários estiveram em férias, com atestados médicos, afastados ou que se recusaram a responder foram excluídos da amostra.

\section{Instrumentos}

O instrumento de coleta de dados foi um questionário de autopreenchimento, contendo dados referentes a idade, escolaridade, tempo de serviço na instituição e se o respondente exercia um cargo de liderança. Ainda, esse questionário continha quatro escalas que avaliaram as variáveis da pesquisa.

\section{Escala de percepção de oportunidades de aprendizagem nas organizações (EPOA)}

A ferramenta tem o objetivo de verificar a percepção de oportunidades de aprendizagem formal e informal que os funcionários têm em suas organizações. A escala possui 13 frases que o funcionário precisa avaliar em relação a sua concordância ou discordância de cada item, por meio de uma escala de 0 (discorda totalmente) a 10 (concorda totalmente). O processo de adaptação, validação e simplificação do instrumento foi realizado por Mourão et al. (2012) e a escala original foi construída por Del Maestro Filho (2004).

\section{Escala de avaliação do estilo gerencial (EAEG)}

A ferramenta foi construída e validada por Melo (2004), em Brasília (DF), e tem como objetivo avaliar os estilos gerenciais adotados pelos gerentes na organização. $O$ instrumento possui três dimensões, com seus respectivos itens de avaliação: tarefa (6 itens), relacionamento (9 itens) e situação (4 itens). Dessa forma, a escala tem, no total, 19 itens que descrevem comportamentos apresentados pelas chefias no dia a dia de trabalho com seus subordinados, todos com sentido positivo, devendo o respondente indicar o quanto cada um reflete a maneira como seu chefe age na organização.

\section{Escala de intenção de rotatividade (EIR)}

A ferramenta pretende avaliar o grau em que um indivíduo elabora planos sobre sua saída da empresa. A escala é composta por três frases que levam o trabalhador a declarar o quanto ele pensa, planeja e tem vontade de, no futuro, deixar de trabalhar na empresa com a qual mantém vínculo formal de trabalho. Siqueira, Gomide, Moura e Marques (1997), submeteram a medida a um novo processo de validação fatorial, em 2014, para que pudessem ser confirmadas suas características psicométricas (Siqueira, 2014).

\section{Inventário de capital psicológico no trabalho (ICPT-12)}

A escala foi originalmente construída e validada por Luthans et al. (2007) e adaptada para o Brasil, na sua forma reduzida, por Siqueira et al. (2011). Constitui-se de uma escala unifatorial, composta por 12 frases. As respostas foram dadas numa escala de cinco pontos, tipo Likert $(1=$ discordo totalmente; $5=$ concordo totalmente). O conceito de capital psicológico no trabalho, medido pelo instrumento, é definido por Siqueira (2014) como o estado mental positivo no qual se articulam quatro categorias de crenças que auxiliam o indivíduo em seu ambiente de trabalho a ver possibilidades de êxito (eficácia), a acreditar em fatos positivos ocorrendo futuramente (otimismo), a ter convicção de que dispõe de recursos (agency) e meios (pathways) para ser bem-sucedido (esperança), e de que sairá fortalecido ao enfrentar adversidades (resiliência) (Siqueira, 2014).

\section{Procedimentos}

A coleta dos dados foi realizada numa instituição de ensino superior privada, comunitária e filantrópica, por meio do contato com os gestores responsáveis pelas equipes, explicando a proposta de pesquisa e solicitando a autorização para liberação dos funcionários. Posteriormente, os funcionários foram convidados a se dirigir a uma sala para participar da pesquisa. Os questionários e as instruções sobre eles, além do termo de consentimento, foram entregues por alunos previamente treinados e capacitados para realizar a tarefa.

\section{Análise dos dados}

Após a codificação dos instrumentos, foi feita uma dupla entrada dos dados no programa EpiData Software 3.1., para checagem automática durante a digitação. Os dados foram convertidos para o programa Statistical Package for the Social Sciences (SPSS, IBMCorp., Somers, NY, USA) versão 21.0.

A análise univariada foi realizada a fim de se obter as frequências simples de todas as variáveis categóricas. Para as variáveis numéricas, foram obtidas as medidas de tendência central e dispersão. Para a análise bruta, foi empregado o teste t de Student, e para a análise multivariada foi utilizada a regressão 
linear múltipla. Em todas as análises foi utilizado um nível de significância de 0,05.

\section{Resultados}

A Tabela 1 mostra o perfil de uma população de técnicos administrativos $(\mathrm{n}=102)$ de uma universidade comunitária e privada do Brasil. Verifica-se que a idade média da população é de 39,6 anos, alinhada com tempo médio de serviço de 11 anos, revelando uma equipe madura e que permanece por um longo período na empresa. Em relação à escolaridade, $24,5 \%$ possuem pós-graduação, 44,1\% o ensino superior completo ou incompleto, $20,6 \%$ têm o ensino médio completo e $8,8 \%$ o ensino fundamental completo ou incompleto. Por fim, 13,7\% da população desempenhava o papel de gestor. O número amostral, inferior a 102, encontrado em algumas variáveis, se deveu ao não preenchimento dessas variáveis por alguns funcionários.

\section{Tabela 1}

Distribuição quanto a idade (média \pm DP), escolaridade (\%), tempo de serviço (média \pm DP) e papel de liderança (\%) dos funcionários técnicoadministrativos.

\begin{tabular}{lcc}
\hline & $\mathbf{N}$ & $\begin{array}{c}\text { \% ou } \\
\text { Média } \pm \text { DP }\end{array}$ \\
\hline Idade & 100 & $39,6 \pm 12,1$ \\
\hline Escolaridade & 100 & \\
\hline $\begin{array}{l}\text { Ensino fundamental } \\
\text { incompleto ou completo }\end{array}$ & 9 & 8,8 \\
Ensino médio completo & 21 & 20,6 \\
Ensino superior incompleto & 21 & 20,6 \\
Ensino superior completo & 24 & 23,5 \\
Pós-graduação & 25 & 24,5 \\
\hline Tempo de serviço & 100 & 132,0 meses \\
& & $(11$ anos $\pm 123,5$ \\
\hline Exerce papel de gestor & 102 & \\
atualmente & 14 & 13,7 \\
\hline Sim & 88 & 86,3 \\
Não &
\end{tabular}

A Tabela 2 apresenta, em média e desvio-padrão, as respostas dos funcionários técnico-administrativos em relação aos instrumentos de avaliação. Na escala EPOA, os resultados retratam uma percepção de oportunidades de aprendizagem mediana por parte dos funcionários, com uma média de $6,7( \pm 1,85)$, num total de 10 pontos.
No ICPT foi encontrada uma média de $3,9( \pm 0,55)$, sugerindo, assim, que os funcionários possuem uma crença média no que se refere a sua esperança, resiliência, otimismo e eficácia no ambiente de trabalho. A EIR revelou uma média de 2,3 $( \pm 1,1)$, manifestando uma baixa intenção dos funcionários técnico-administrativos em sair da universidade.

Em relação ao EAEG, a Tabela 2 mostra os três domínios, em que o estilo voltado ao relacionamento apresentou uma média de $4,2( \pm 0,73)$, o voltado à tarefa $4,2( \pm 0,61)$ e o voltado à situação $4,2( \pm 0,71)$. Isto é, os funcionários identificam os três perfis gerenciais na instituição.

\section{Tabela 2}

Distribuição das respostas dos funcionários técnicoadministrativos em relação aos instrumentos de avaliação.

\begin{tabular}{lcc}
\hline Instrumento & $\mathbf{N}$ & Média $\pm \mathbf{D P}$ \\
\hline EPOA1 & 91 & $6,7 \pm 1,85$ \\
ICPT2 & 100 & $3,9 \pm 0,55$ \\
EIR3 & 102 & $2,3 \pm 1,1$ \\
EAEG4 & & \\
Relacionamento & 95 & $4,2 \pm 0,73$ \\
Tarefa & 100 & $4,2 \pm 0,61$ \\
Situacional & 101 & $4,2 \pm 0,71$ \\
\hline
\end{tabular}

\footnotetext{
${ }^{1} \mathrm{O}$ instrumento utiliza uma escala de 0 a 10 , perfazendo um total de 10 pontos. Valores médios entre 0 e 4 indicam percepção de que as oportunidades são escassas; entre 4,1 e 7, percepção de que a organização oferece oportunidades medianas de aprendizagem; e entre 7,1 e 10, percepção de que a organização incentiva a aprendizagem formal e informal.

${ }^{2} \mathrm{O}$ instrumento utiliza uma escala de 1 a 5 , na qual os resultados entre 5 e 4 indicam altos escores; iguais a 3 indicam médio; e inferior ou igual a 2 indicam baixo capital psicológico no trabalho. Assim, quanto maior o valor do escore médio, mais fortalecidas são as crenças do trabalho no que se refere a sua esperança, resiliência, otimismo e eficácia no ambiente de trabalho.

${ }^{3} \mathrm{O}$ instrumento utiliza uma escala de 1 a 5 . Valores médios entre 1 e 2,9 indicam menor intenção de rotatividade; entre 3 e 3,9, média intenção de rotatividade; e entre 4 e 5, alta intenção de rotatividade.

${ }^{4} \mathrm{O}$ instrumento utiliza uma escala de 1 a 5 , na qual resultados superiores ao ponto médio, que corresponde ao valor 3 , indicam estilo gerencial mais voltado ao relacionamento, tarefa ou situação.
} 
Psicologia: Ciência e Profissão 2021 v. 41, e219584, 1-12.

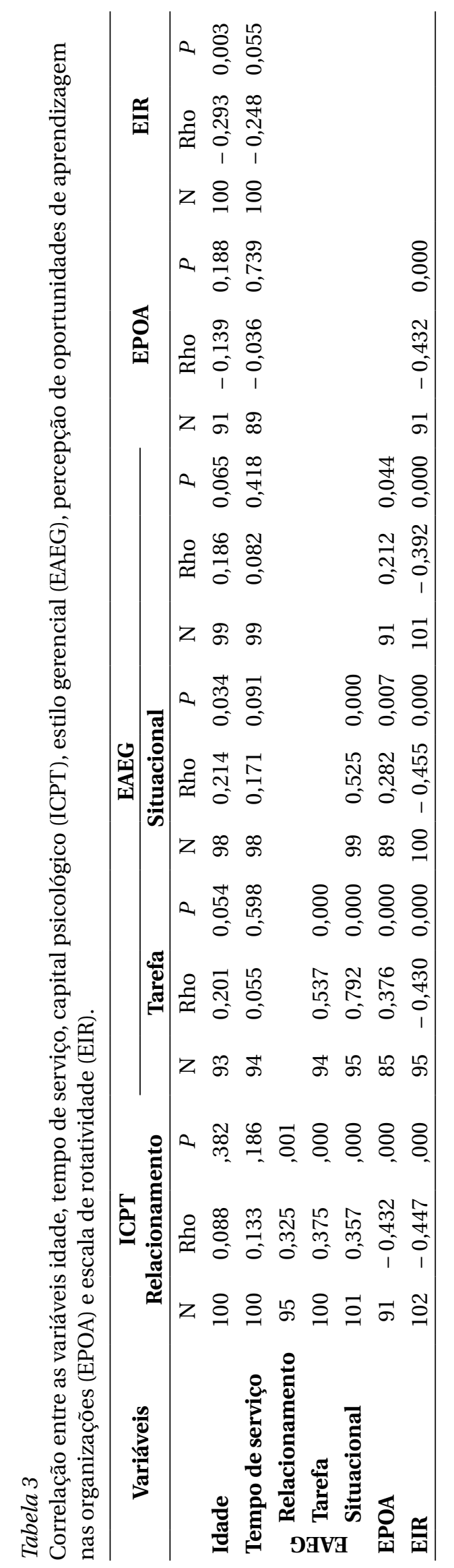


A Tabela 3 apresenta as correlações entre as variáveis testadas, que serão descritas de acordo com a significância alcançada. Assim sendo, foi identificada uma correlação positiva entre a idade e o estilo gerencial voltado para tarefa $(\mathrm{p}=0,034)$, em que quanto maior a idade dos entrevistados, maior a identificação com o estilo gerencial focado na tarefa. Ainda, observa-se que quanto maior a idade, menor a intenção de rotatividade dos funcionários técnico-administrativos no âmbito universitário ( $\mathrm{p}=0,003)$.

Com relação ao capital psicológico, foi identificada uma correlação positiva com os estilos de liderança, em que quanto maior o estilo de relacionamento $(\mathrm{p}=0,001)$, tarefa $(\mathrm{p}=0,000)$ e situacional $(\mathrm{p}=0,000)$ do gestor, maior a identificação do funcionário como um capital psicológico no trabalho. Também foram significativos os dados sobre as oportunidades $(p=0,000)$, segundo os quais quanto menor a percepção dos funcionários acerca das oportunidades de aprendizagem, maior a identificação com o capital psicológico no trabalho. Além disso, identificou-se uma correlação negativa com a escala de rotatividade, na qual quanto mais o funcionário se percebe como capital psicológico, menor seu interesse em sair da empresa $(\mathrm{p}=0,000)$.

Com relação às oportunidades de aprendizagem e estilo gerencial, foi verificada uma correlação positiva com o estilo gerencial de relacionamento $(p=0,000)$, tarefa $(\mathrm{p}=0,007)$ e situacional $(\mathrm{p}=0,044)$ por parte dos gestores. Sobre a intenção de rotatividade, o funcionário que se percebe com maior oportunidade de aprendizagem tem menor intenção de sair da instituição $(p=0,000)$, assim como quanto melhor a constatação do funcionário do estilo de relacionamento $(\mathrm{p}=0,000)$, tarefa $(\mathrm{p}=0,000)$ e situacional $(\mathrm{p}=0,000)$ avaliado frente ao gestor, menor sua intenção de rotatividade.

\section{Discussão}

A partir dos resultados obtidos, identifica-se uma equipe madura e que permanece por um longo período na empresa. Revela-se também um alto percentil de qualificação técnica, no qual a maioria $(68,6 \%)$ dos funcionários possui, no mínimo, ensino superior incompleto e 86,3\% não exercem papel de gestor.

Em vista do papel estratégico do processo de aprendizagem nas empresas, papel que se estende ao âmbito universitário, em especial às instituições de ensino superior, é necessário um constante investimento no aperfeiçoamento dos funcionários técnico-administrativos que compõem os alicerces institucionais. Nesse sentido, este trabalho demonstrou um escore de 6,7 na percepção dos funcionários relativa às oportunidades de aprendizagem. Esse resultado é identificado de forma positiva, em vista do cenário econômico do Brasil, onde as instituições de ensino são obrigadas a reajustar os gastos com investimentos referentes à capacitação. Dessa forma, há uma predominância de capacitações internas ou por meio de parceiros institucionais, em detrimento do investimento em capacitações externas, reduzindo-se assim os custos para a organização.

Por intermédio da identificação de uma abordagem positiva do trabalho (Psicologia Positiva), que visa o desenvolvimento das pessoas e das capacidades psicológicas positivas dos funcionários, com possíveis aplicações diretas na gestão do desempenho em instituições, pode-se considerar que os gestores, nesse espaço, possuem uma responsabilidade como agentes de mudança, influenciando diretamente os processos de aprendizagem. Os resultados desta revelam não haver diferença de média $(4,2)$ entre os três estilos gerenciais (relacionamento, tarefa e situacional), denotando que os funcionários identificam os três estilos gerenciais dentre os gestores. Esse dado pode ser interpretado sob dois aspectos: o primeiro refere-se a diferenças na maturidade entre as áreas, gestores e equipes e o direcionamento da instituição em se desenvolver de acordo com as necessidades identificadas. Quer dizer, em áreas que ainda necessitam de maior desenvolvimento técnico, nas definições de padrões, procedimentos e métodos, o gestor ainda atua com foco na tarefa; em áreas que já possuem processos definidos, os gestores valorizam o relacionamento e a confiança mútua; por fim, há os setores que oscilam de acordo com a realidade do ambiente de trabalho momentâneo. O segundo aspecto pode retratar uma falta de hegemonia entre os gestores, o que é possivelmente uma falta de capacitação permanente da liderança e de direcionamento da área de gestão de pessoas no que se refere ao padrão ideal de líder para a instituição. A referência bibliográfica indica que o estilo gerencial de relacionamento e tarefa são voltados para a aquisição e compartilhamento de conhecimentos, que favorecem a aprendizagem, o que reforça os achados desta pesquisa referentes às oportunidades de aprendizagem organizacional e ao estilo de liderança (Isidoro-Filho \& Guimarães, 2008).

Nesse contexto, o psicólogo positivo Luthans et al. (2010) propôs um novo olhar para a força de trabalho, 
dando ênfase ao trabalhador de forma individual, por meio do capital psicológico. De acordo com Siqueira (2014), o capital psicológico possui quatro capacidades psicológicas positivas: autoeficácia (convicções acerca de habilidades para mobilizar recursos cognitivos ou cursos de ação necessários para executar com sucesso uma tarefa específica em um dado contexto); otimismo (pessoas que esperam que coisas boas ocorram com elas); esperança (estado motivacional positivo baseado em um senso de sucesso e em um plano para atingir metas); e resiliência (habilidade psicológica positiva que faz alguém acometido por uma adversidade reagir e conseguir se sobressair e vencer tais situações).

Neste trabalho, o escore médio de 3,9 referente ao instrumento que verifica o capital psicológico no trabalho indica que os funcionários possuem uma crença média quanto a sua esperança, resiliência, otimismo e eficácia no ambiente profissional. De acordo com a pesquisa realizada por Avey, Reichard, Luthans e Mhatre (2011), quanto maior o escore, maior é a relação positiva e significativa com atitudes, comportamentos e desempenho dos funcionários, como a satisfação no trabalho, o comprometimento organizacional, o bem-estar psicológico, o comportamento de cidadania organizacional e as medidas de desempenho no trabalho.

Ao relacionar os dados sobre a identificação do capital psicológico e os estilos de liderança, identifica-se uma correlação positiva entre ambos, nos três estilos gerenciais. Ou seja, os resultados indicam que qualquer estilo de liderança pode promover capital psicológico na instituição avaliada. Esses resultados corroboram os achados referentes ao escore médio do estilo gerencial, em que os três estilos obtiveram os mesmos escores. No mesmo sentido, o estudo realizado por Isidro-Filho e Guimarães (2008), que também utilizou o instrumento EAEG, demonstrou que, em geral, os funcionários percebem que seus líderes buscam comportar-se de forma orientada ao relacionamento com seus subordinados e agem de modo voltado a indicar tarefas, normas e regras para o grupo, além de valorizar o respeito à autoridade, à disciplina e à hierarquia. Além disso, o estudo identificou que os funcionários percebem que seus líderes oferecem liberdade de trabalho em função da competência, motivação, segurança e disposição de seus subordinados. Assim, os resultados indicam uma correta alocação dos perfis de liderança nos diferentes setores, sendo compatíveis com as necessidades de cada setor, promovendo, portanto, o capital psicológico dos funcionários.

Em contrapartida, foi identificada uma correlação negativa entre capital psicológico e a percepção de oportunidade. Ou seja, os funcionários identificados com maior capital psicológico no trabalho percebem uma baixa oportunidade de aprendizado, o que é contraditório, uma vez que pesquisas têm mostrado que a percepção de oportunidades de aprendizagem está relacionada a comportamentos organizacionais como cidadania, comprometimento e bem-estar no trabalho. Acredita-se que essa falta de correlação positiva entre ambos os aspectos seja um reflexo da dificuldade de investimento em capacitação por parte das organizações de ensino, o que, no entanto, parece não atingir, até o momento, o capital psicológico dos funcionários. Conforme trabalho realizado por Comparsi (2018), somente a implementação de ações de TD\&E não se mostra suficiente para impactar positivamente os processos organizacionais. Tendo em vista que a percepção de oportunidades por um funcionário tende a motivar positivamente a realização de atividades de empregabilidade, como aquisição de novos conhecimentos e novas habilidades, sugere-se um treinamento mais focado nas competências e conteúdos condizentes com o dia a dia do funcionário, aplicado à especificidade da instituição, com o objetivo de estabelecer resultados voltados à formação de vínculo entre a instituição e o funcionário e melhorar sua atuação.

Com relação à escala de rotatividade, os resultados demonstram uma baixa intenção dos funcionários técnico-administrativos em sair da universidade. Da mesma forma, foi identificado que quanto maior o capital psicológico, menor é a intenção de rotatividade do funcionário. A literatura aponta que a menor intenção de sair da empresa geralmente está correlacionada à satisfação no trabalho, ao clima organizacional positivo e ao comprometimento organizacional, fatores desencadeantes de atitudes positivas no funcionário em relação à organização da empresa. Assim, sugere-se uma relação positiva entre as dimensões utilizadas para analisar intenção de rotatividade, satisfação e comprometimento organizacional, e as dimensões do capital psicológico, autoeficácia, otimismo, esperança e resiliência, de acordo com um trabalho realizado por Ponte (2018).

Segundo Polizzi Filho e Siqueira (2012), em estudo desenvolvido com professores universitários no Brasil, quanto maior o comprometimento do professor com a escola, menor a intenção de rotatividade. Ademais, 
em 2019, Polizzi Filho e Claro descrevem a moderação do capital psicológico sobre o bem-estar no trabalho e a intenção de rotatividade por meio do comprometimento afetivo por parte dos profissionais com a instituição. Não obstante, a implementação de técnicas voltadas ao treinamento dos funcionários influencia diretamente a capacidade da empresa de manter profissionais qualificados. Estudos que Steil, Dandolini, Souza, Cuffa e Costa (2018) e Steil, Cuffa, Iwaya e Pacheco (2020) realizaram no Brasil apresentaram uma correlação negativa entre a percepção de oportunidades de aprendizagem e a rotatividade entre trabalhadores, demonstrando a necessidade de ações focadas em promover a aprendizagem dos funcionários além da valorização, por parte dos empregados, nas ações oferecidas, para manter a empregabilidade.

No que tange ao estilo gerencial, este trabalho mostrou que independente do perfil de liderança (relacionamento, tarefa ou situacional), há uma baixa intenção de rotatividade. Esse resultado denota a importância da relação entre gestor e funcionário para a retenção dos trabalhadores na empresa. Assim, independentemente do estilo gerencial, com foco no relacionamento, tarefa ou situação, a relação impacta a tomada de decisão do funcionário em buscar outras oportunidades fora da empresa. No entanto, de acordo com os achados de Fonseca, Porto e Borges-Andrade (2015), em um trabalho sobre a produção científica brasileira sobre liderança, há pouco interesse em pesquisar o impacto da liderança em melhores resultados, como satisfação, comprometimento e desempenho da equipe. Mas, de acordo com os autores, há sinais de efeitos do perfil de liderança nas relações de confiança entre gestor e funcionário.

Ainda, foi observada uma correlação entre a intenção de rotatividade e a idade mais baixa dos funcionários, corroborando os resultados apresentados por Cappi e Araújo (2015) sobre satisfação no trabalho, comprometimento organizacional e intenção de sair entre as gerações $\mathrm{Y}$ (trabalhadores jovens) e X (trabalhadores com idade avançada). Os autores indicam que a geração $X$ atinge maior maturidade e estabilidade profissional, de forma que são considerados mais satisfeitos e comprometidos, possuindo menor intenção de sair da empresa.

Outra sugestão de análise é o atual momento de instabilidade financeira do Brasil e o receio de não ser absorvido pelo mercado de trabalho em virtude da remuneração e da idade mais avançada. Ainda sobre a variável idade, existe uma relação significativa entre a idade mais avançada e a identificação do estilo gerencial voltado para a tarefa, que, de acordo com o estudo de Cavazotte, Lemos e Viana (2012), pode traduzir também o perfil dos funcionários da geração $\mathrm{X}$, com características de individualismo e oportunismo (Cappi \& Araujo, 2015). Dessa forma, os autores provocam outros pesquisadores a traduzirem a linguagem científica em linguagem comum, de modo que os trabalhos científicos possam contribuir com estratégias e processos de melhorias para empresas e pessoas e, assim, possa ocorrer o amadurecimento e fortalecimento da área de gestão. Além disso, é esperado nas práticas de gestão de pessoas que a identificação de desenvolvimento por parte dos funcionários fortaleça a relação com a empresa e crie um ambiente de crescimento, satisfação e retenção de talentos.

\section{Conclusão}

Assim sendo, este trabalho identificou que os funcionários técnico-administrativos de um ambiente universitário percebem uma média oportunidade de aprendizado, que parece estar ligada a um aumento do capital psicológico, refletindo em uma baixa intenção de rotatividade. Dessa forma, a busca constante pela identificação da realidade institucional, referente às expectativas dos funcionários, deve ser uma constante, permitindo promover ações estratégicas de gestão de pessoas focadas no desenvolvimento e retenção, contribuindo, consequentemente, para o desenvolvimento das universidades.

\section{Referências}

Avey, J. B., Luthans, F., \& Youssef, C. M. (2010). The additive value of psychological capital in predicting work attitudes and behaviors. Journal of Management, 36(2), 430-452. https://doi.org/10.1177/0149206308329961

Avey, J. B., Reichard, R. J., Luthans, F., \& Mhatre, K. H. (2011). Meta-analysis of the impact of positive psychological capital on employee attitudes, behaviors, and performance. Human Resource Development Quarterly, 22(2), 127-152. https://doi.org/10.1002/hrdq.20070 
Cappi, M. N., \& Araujo, B. F. B. (2015). Satisfação no trabalho, comprometimento organizacional e intenção de sair: Um estudo entre as gerações X e Y. Revista Eletrônica de Administração, 21(3), 576-600. https://doi.org/ $10.1590 / 1413-2311.0782014 .54843$

Carmo, G. (2009). Antecedentes da intenção de rotatividade: Comprometimento organizacional e confiança do empregado na organização [Dissertação de Mestrado, Universidade Federal de Uberlândia]. Repositório Institucional UFU. http://www.pgpsi.ip.ufu.br/node/172

Cavazotte, F. S. N., Lemos, A. H. C., \&Viana, M. D. A. (2012). Novas gerações no mercado de trabalho: Expectativas renovadas ou antigos ideais? Cadernos EBAPE.BR, 10(1), 162-180. https://doi.org/10.1590/S1679-39512012000100011

Chiuzi, R. M., Siqueira, M. M. M., \& Martins, M. C. F. (2012). As dimensões da organização positiva e seus impactos sobre o bem-estar dos trabalhadores. Mudanças: Psicologia da Saúde, 20(1-2), 31-40. https://doi. org/10.15603/2176-1019/mud.v20n1-2p31-40

Coelho Jr., F. A. (2011). Suporte à aprendizagem informal no trabalho: Uma proposta de articulação conceitual. Revista de Administração Mackenzie, 12(6), 224-253. https://doi.org/10.1590/S1678-69712011000600010

Comparsi, A. W. (2018). Processos de aprendizagem formais e informais em instituições públicas [Monografia, Universidade Federal do Rio Grande do Sul]. Repositório Digital UFRGS. https://lume.ufrgs.br/handle/ $10183 / 185003$

Del Maestro Filho, A. (2004). Modelo relacional entre modernização organizacional, práticas inovadoras de treinamento e satisfação no trabalho. [Tese de Doutorado, Universidade Federal de Minas Gerais]. Repositório Digital UFMG. http://hdl.handle.net/1843/BUBD-99UFM7

Fonseca, A. M. O., Porto, J. B., \& Borges-Andrade, J. E. (2015). Liderança: Um retrato da produção científica brasileira. Revista de Administração Contemporânea, 19(3), 290-310. https:// doi.org/10.1590/1982-7849rac20151404

Isidro-Filho, A., \& Guimarães, T. A. (2008). Estilos de liderança e mecanismos de aprendizagem em organizações: Análises de suas relações em uma organização financeira. Revista Eletrônica de Gestão Organizacional, 6(3), 361378. https://doi.org/10.51359/1679-1827.2008.21531

Lacerda, E. R. M., \& Abbad, G. (2003). Impacto do treinamento no trabalho: Investigando variáveis motivacionais e organizacionais como suas preditoras. Revista de Administração Contemporânea, 7(4), 77-96. https://doi. org/10.1590/S1415-65552003000400005

Luthans, F, Avey, J. B., Avolio, B. J., \& Peterson, S. J. (2010). The development and resulting performance impact of positive psychological capital. Human Resource Development Quarterly, 21(1), 41-67. https://doi.org/10.1002/ hrdq.20034

Luthans, F., Youssef, C., \& Avolio, B. (2007). Psychological capital: Developing the human competitive edge. Oxford University Press.

Melo, E. A. A. (2004). Escala de avaliação do estilo gerencial (EAEG): Desenvolvimento e validação. Revista Psicologia: Organização e Trabalho, 4(2), 31-62.

Mourão, L., \& Meneses, P. P. M. (2012). Medidas de impacto de TD\&E: O nível do valor final. In G. S. Abbad, L. Mourão, P. P. M. Meneses, T. Zerbini, J. E. Borges-Andrade, \& R. Dias (Orgs.), Medidas de Avaliação em TD\&E. (pp. 177-188). Artmed.

Perryer, C., Jordan, C., Firns, I., \& Travaglione, A. (2010). Predicting turnover intentions: The interactive effects of organizational commitment and perceived organizational support. Management Research Review, 33(9), 911-923. https://doi.org/10.1108/01409171011070323

Picoli,F.R., \&Takahashi,A. (2016).Capacidadedeabsorção, aprendizagemorganizacional emecanismos deintegração social. Revista de Administração Contemporânea, 20(1), 1-20. https://doi.org/10.1590/1982-7849rac2016140036

Polizzi Filho, A., \& Claro, J. A. C. S. (2019). The impact of well-being at work and psychological capital over the turnover intention: A study of teachers. Revista de Administração Mackenzie, 20(2), 1-27. https://doi. org/10.1590/1678-6971/eRAMG190064

Polizzi Filho, A., \& Siqueira, M. M. M. (2012). O impacto de bem-estar no trabalho e de capital psicológico sobre intenção de rotatividade: Um estudo com professores [Trabalho apresentado]. Congresso Nacional de Psicologia da Saúde, Promoção da Saúde e Doenças Crônicas: Desafios à Promoção da Saúde, Brasil. 
Ponte, L. A. (2018). Relações entre capital psicológico e intenção de rotatividade: Um estudo no restaurante Cabaña del Primo Riomar [Monografia, Universidade Federal do Ceará]. Repositório UFC. http://www.repositorio.ufc. $\mathrm{br} /$ handle/riufc/38752

Santos Filho, G. M., \& Mourão, L. (2011). A relação entre comprometimento organizacional e impacto do treinamento no trabalho. Revista Psicologia: Organizações e Trabalho, 11(1), 75-89.

Siqueira, M. M. M. (2014). Novas medidas do comportamento organizacional: Ferramentas de diagnóstico e de gestão. Artmed.

Siqueira M. M. M., Gomide, J., Moura, O. I., \& Marques, T. M. (1997). Um modelo pós-cognitivo para intenção de rotatividade: Antecedentes afetivos e cognitivos [Trabalho apresentado]. XXVI Congresso Interamericano de Psicologia, São Paulo, SP, Brasil.

Steil, A. V., Cuffa, D., Iwaya, G. H., \& Pacheco, R. C. S. (2020). Perceived learning opportunities, behavioral intentions and employee retention in technology organizations. Journal of Workplace Learning, 32(2), 147-159. https:// doi.org/10.1108/JWL-04-2019-0045

Steil, A. V., Dandolini, G. A., Souza, J. A., Cuffa, D., \& Costa, R. (2018). Behavioral intentions and retention of technical and scientific staff in research and development organizations. International Journal of Human Capital and Information Technology Professionals, 9(2), 16-31. https://doi.org/10.4018/IJHCITP.2018040102

Veloso, A. L. O. M., Silva, M. J., Silva, I., \& Caetano, A. (2015). Fatores que afetam a transferência da aprendizagem para o local de trabalho. Revista de Administração de Empresas, 55(2), 188-201. https://doi.org/10.1590/ S0034-759020150208

\section{Juliana de Araújo Germano}

Mestre em Saúde e Comportamento pela Universidade Católica de Pelotas (UCPel), Pelotas - RS. Brasil.

Graduação em Psicologia na Universidade Católica de Pelotas (UCPel), Pelotas - RS. Brasil.

Especialista em Gestão Estratégica de Pessoas pela Fundação Getúlio Vargas (FGV), São Paulo - SP. Brasil.

E-mail: juagermano@hotmail.com

(D) https://orcid.org/0000-0003-2412-6698

\section{Cainá Corrêa do Amaral}

Mestranda em Saúde e Comportamento e bacharel em Enfermagem pela Universidade Católica de Pelotas (UCPel), Pelotas - RS. Brasil.

E-mail: caina.ca@hotmail.com

(1) https://orcid.org/0000-0002-5603-5734

\section{Tiago Fernandez Garcia}

Graduando em Fisioterapia na UCPel, Pelotas - RS. Brasil.

E-mail: tiagogarcia.fisio@gmail.com

(1) https:// orcid.org/0000-0002-1951-3418

\section{Geovanna Peter Corrêa}

Graduanda em Fisioterapia na UCPel, Pelotas - RS. Brasil.

E-mail: geovannapeter_@live.com

(1) https://orcid.org/ 0000-0002-6397-9127

\section{Camila Perelló Ferrúa}

Doutoranda em Saúde e Comportamento e mestre em Odontologia pela UCPel, Pelotas - RS. Brasil.

E-mail: camila_perello@hotmail.com

(1) https://orcid.org/0000-0001-8071-377X 


\section{Luciana de Ávila Quevedo}

Professora adjunta da UCPel. Doutora e mestre em Saúde e Comportamento pela UCPel, Pelotas - RS. Brasil.

E-mail: lu.quevedo@bol.com.br

(1) https://orcid.org/0000-0001-6883-4333

Fernanda Nedel

Professora adjunta da UCPel. Doutora em Biotecnologia pela UFPel, Pelotas - RS. Brasil.

E-mail: fernanda.nedel@gmail.com

(1) https://orcid.org/0000-0002-9055-8328

Endereço para envio de correspondência:

Universidade Católica de Pelotas. Programa de Pós-Graduação em Saúde e Comportamento. Rua Gonçalves Chaves, 377, sala 411, prédio C, Centro. CEP: 96015-560. Pelotas - RS. Brasil.

Recebido 06/02/2019

Aceito 21/12/2020

Received 02/06/2019

Approved 12/21/2020

Recibido 06/02/2019

Aceptado 21/12/2020

Como citar: Germano, J. A., Amaral, C. C., Garcia, T. F., Corrêa, G. P., Ferrúa, C. P., Quevedo, L. A., \& Nedel, F. (2021). Percepção de oportunidades de aprendizado de funcionários técnico-administrativos no âmbito universitário. Psicologia: Ciência e Profissão, 41, 1-12. https://doi.org/10.1590/1982-3703003219584

How to cite: Germano, J. A., Amaral, C. C., Garcia, T. F., Corrêa, G. P., Ferrúa, C. P., Quevedo, L. A., \& Nedel, F. (2021). Perception learning opportunities of technical-administrative staff in the university scope. Psicologia: Ciência e Profissão, 41, 1-12. https://doi.org/10.1590/1982-3703003219584

Cómo citar: Germano, J. A., Amaral, C. C., Garcia, T. F., Corrêa, G. P., Ferrúa, C. P., Quevedo, L. A., \& Nedel, F. (2021). Percepción del personal técnico y administrativo sobre las oportunidades de aprendizaje en las universidades. Psicologia: Ciência e Profissão, 41, 1-12. https:// doi.org/10.1590/1982-3703003219584 